\section{Errata and Addendum for "Vertical Mixing in the Fully Developed Turbulent Layer of Sediment-Laden Open-Channel Flow" by E. A. Toorman}

September 2008, Vol. 134, No. 9, 1225-1235.

DOI: 10.1061/(ASCE)0733-9429(2008)134:9(1225)

\section{Erik A. Toorman}

Hydraulics Laboratory, Civil Engineering Dept., Katholieke Universiteit Leuven, Kasteelpark Arenberg 40, B-3001 Heverlee, Belgium. E-mail: erik.toorman@bwk.kuleuven.be

The factor $\alpha$ in the last term between brackets in Eqs. (30) and (31) should not be there, since it cancels out during the manipulations. This remained unnoticed because the value of $\alpha$ in the validation is 1 . Hence, the corrected equations should read

$$
\begin{aligned}
\overline{v_{z}^{\prime} \phi^{\prime}} & =\left(\beta_{0} v_{t}-\tau_{t} w_{s}^{2}\right) \frac{\partial \bar{\phi}}{\partial z}+\beta_{0} \tau_{t} \bar{\phi} \frac{\partial}{\partial z}\left(\frac{v_{t}}{\tau_{t}}\right) \\
& =\beta_{0} v_{t}\left(\left(1-\frac{\alpha}{\beta_{0} c_{\mu}} \frac{w_{s}^{2}}{k}\right) \frac{\partial \bar{\phi}}{\partial z}+\frac{\bar{\phi}}{k} \frac{\partial k}{\partial z}\right)
\end{aligned}
$$

$$
\beta_{0} S c_{t}=\left(1-\frac{\alpha}{\beta_{0} c_{\mu}} \frac{w_{s}^{2}}{k}+\frac{\frac{\partial \ln k}{\partial z}}{\frac{\partial \ln \bar{\phi}}{\partial z}}\right)^{-1}=\left(1-\frac{\alpha}{\beta_{0} c_{\mu}} \frac{w_{s}^{2}}{k}+\frac{\partial \ln k}{\partial \ln \bar{\phi}}\right)^{-1}
$$

In addition, it should be noticed that the concentration dependence of the turbulent Schmidt number is only apparent. The concentration in the turbophoresis term can be eliminated by the use of the flux balance, Eq. 1 in the original paper with the opposite sign convention for $w_{s}$, as in the remainder of the manuscript. The eddy diffusivity is then replaced by the eddy viscosity divided by the turbulent Schmidt number and the turbulent Schmidt number is then obtained after rearrangement of the equation, resulting in

$$
\frac{S c_{t}}{S c_{0}}=\frac{\left(1+\beta_{0} \frac{v_{t}}{w_{s} k} \frac{\partial k}{\partial z}\right)}{\left(1-\frac{\alpha}{\beta_{0} c_{\mu}} \frac{w_{s}^{2}}{k}\right)}=\frac{\left(1+\frac{\beta_{0} c_{\mu}}{\alpha} \frac{\tau_{t}}{w_{s}} \frac{\partial k}{\partial z}\right)}{\left(1-\frac{\alpha}{\beta_{0} c_{\mu}} \frac{w_{s}^{2}}{k}\right)}
$$

and: 